\title{
Systems control in railway transport, challenges towards managing integration
}

\author{
Merishna Ramtahalsing ${ }^{\mathrm{a}^{*}}$, Willem Haanstra ${ }^{\mathrm{a}}$, Jan Braaksma ${ }^{\mathrm{a}}$, Mohammad \\ Rajabalinejad $^{\mathrm{a}}$, Leo van Dongen ${ }^{\mathrm{a}}$
}

${ }^{a}$ Department of Design Production \& Management, University of Twente, De Horst 2, 7522 LW Enschede, The Netherlands

*Corresponding author. Tel. +31534898520. E-mail address: m.m.ramtahalsing@utwente.nl

\begin{abstract}
The growing demand for optimal rail mobility requires an efficient and well-integrated railway system, one which can cope with technical, logistical, and environmental constraints, to enable sustainable growth. This demand has led to numerous (proposed) changes in railways, including in the Dutch railway system. Because of this, managing system integration is currently a trending topic. Besides technology, systems integration involves humans, organizations, processes, and information sharing. Hence, challenges related to system integration can be manifold.

In order to get insight into these integration challenges in practice, 43 semi-structured exploratory interviews are conducted in the Dutch railway context. In our qualitative data analysis, challenges are analyzed from two frameworks taking a systems perspective. This research reveals numerous challenges faced in this context, most dominant here is the desire to effectively manage changes. Specifically, our analysis and subsequent mapping on systems control theory shows that many of these challenges revolve around effective control in the railway system.

Finally, our research shows that future research should be aimed at gaining early insight and models on changes to be integrated into railway systems. In addition, the findings of this research can be used to help practitioners and researchers to work towards not only recognizing but also resolving the specified integration challenges.
\end{abstract}

Keywords: Challenges; Control; Railways; System Integration; System Performance

\section{Introduction}

Over the last decades, there has been increased attention for seamless railway mobility across borders, especially in Europe. To achieve this mobility within and between countries, modern railway systems are currently adopting technologies like ERTMS (European Rail Traffic Management System) and ATO (Automatic Train Operations) [1, 2]. These recent developments require the integration of systems to improve the functionality and efficiency of the rail system as a whole but are also making these systems increasingly complex.

To deal with these challenges, railway system stakeholders are progressively required to closely collaborate at the inter-organizational-, as well as the international level. In this context, stakeholders are more likely to have diverging views, skills, responsibilities, goals, behavior, and interests. In addition, to achieve interoperability of technical systems, different technical subsystems also have to connect and communicate seamlessly. Hence, different countries, distinct organizations, technical systems, departments, and regulations need to converge on the shared goal of optimal mobility system performance.

All of this requires extraordinary amounts of systems integration (SI) on different levels, and requires functioning within increasingly complex networks with dynamic interconnections and in a changing environment [3].

SI is an omnipresent concept involved in nearly every aspect of the engineering of large systems and systems management [4]. It can be defined as the process of bringing together sub-systems into one system and ensuring that the subsystems function together as a whole $[5,6]$. It is the integration of subsystems and components that gives systems their superiority over a set of elements that do not work together without integration [4]. At its core, SI is a strategic activity that is integral to business management and cuts across technical, management, and strategic levels [6]. Moreover, SI ensures that appropriate communication occurs between technological, human, and organizational elements required to work together. Because of the aforementioned trends, and because systems continue to grow in scale and complexity, managing SI is a key concern within the railway sector.

\subsection{SI as an enabler of System Performance}

All novel and increasingly complex technologies previously mentioned are aimed at improving railway system performance. To do this, these changes need to be integrated into the existing railway context. This

Peer-review under responsibility of the Programme Chair of the 10th International Conference on Through-life Engineering Services. 
requires effective control and management, efficaciously shifting the focus on what needs to be integrated (or not).

The current literature mentions a diverse set of challenges related to SI. For example, Madni \& Sievers [6] mention that (1) integration with legacy systems, (2) lack of common language for communicating among disciplines, and (3) difficulties at interfaces are among these. Moreover, they state that the latter, interfaces, are where nearly all SI failures occur. In their follow-up research, Madni \& Sievers [7] indicate that SI is hampered by (4) the lack of common terminology and (5) clear definitions of technical and nontechnical interdependencies.

Furthermore, current literature pays some attention to SI challenges in railways specifically. I.e. Rajabalinejad [8] mentions that (1) the degree of fragmentation of the rail systems and its interconnectivity, (2) multi-stakeholder nature of decision-making, (3) variety of views of stakeholders, (4) numbers of (revised) rules and regulations, (5) and the arrival of modern technology may impose specific risks to the rail industry.

While SI has been narrowly defined in some industries by rigorous methodologies that achieve specific goals, the suitability of these methods to other industries such as the railway industry is not necessarily seen as practical [6]. In contrast to organizations such as NASA, system cost and integration capacity are limited in the railway sector.

The aforementioned literature mostly positions SI as 'an end in itself' whereas we posit that SI should be regarded more as 'a means to an end'. In order to improve railway systems' performance, changes need to be managed. This position draws attention to which aspects of the system require what level of integration, thus aiding capacity allocation. As such, we investigate SI from the perspectives of safety control and systems control in this article. In order to better understand the SI challenges in the railway context, their respective magnitudes, and to pinpoint the key factors that enable more effective SI, a series of interviews was carried out in the Dutch railway sector.

\section{Methodology}

To explore and identify SI challenges in the railway context, 43 semi-structured exploratory interviews were carried out in the Dutch railway sector and mapped on two consecutive frameworks. This problem-oriented exploration will not only aid practitioners in recognizing these challenges but can also aid researchers and practitioners in finally resolving them.

\subsection{The Dutch railway system}

The Dutch railway system is divided into a core network and peripheral lines [19]. The Dutch rail transport services were privatized in the 1990s and split into independent railway infrastructure manager (ProRail) and main railway operator Netherlands Railways (NS) [9]. This separation of railways infrastructure manager and railway operator means that there is a limit to the improvements that can be achieved if they cannot work together effectively [10].

\subsection{Approach}

The interviews at the infrastructure managing- and main railway operating organization were carried out within different organizational layers, in distinct departments to obtain a comprehensive understanding of SI challenges. Interviewees included: asset managers, change managers, network designers, innovation managers, operation managers, project managers, program managers, safety managers, safety specialists, (systems) engineers, system/infrastructure architects, train drivers, \& quality managers. These interviews were documented and afterward analyzed using qualitative data analysis software ATLAS.ti, the process of which is shown in Figure 1.

This iterative process consists of: (i) conducting the interviews and documenting these; (ii) grouping the interviews based on organization; (iii) manual data processing and coding based on SI issues mentioned during the interviews with help of the software's coding options open-, in-vivo- and list coding; and (iv) structuring and regrouping the analyzed data in order to discover patterns and relationships. This led to (v) a structured analysis of all the data from which (vi) datadriven conclusions could be derived. Moreover, this process has been carried out with multiple researchers to improve the validity of the analysis. From this point on, 'codes' \& 'challenges' will be used interchangeably throughout the paper.

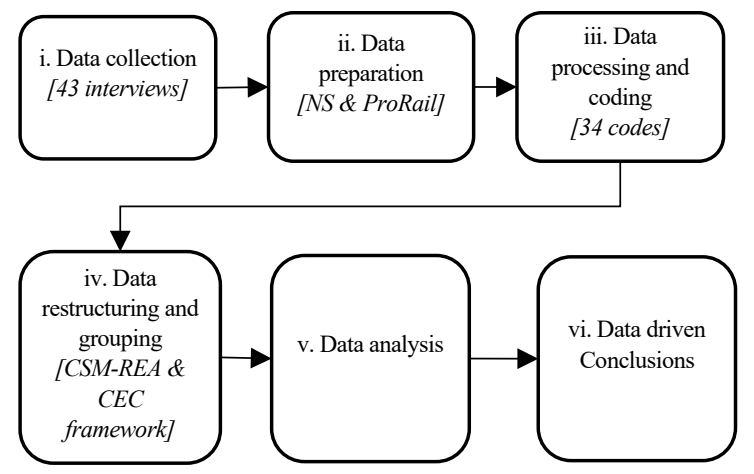

Figure 1. Data analysis process.

To better understand obtained data, the codes were mapped on two different frameworks (step iv). 
Initially, the Common Safety Methods on Risk Evaluation and Assessment (CSM-REA) [11] was applied for data grouping. Subsequently, the 'Conditions for Effective Control' (CEC) framework [12] was used to provide a second data grouping as the CSM-REA did not fully map to the identified codes.

\subsection{Common Safety Methods for Risk Evaluation \& Assessment (CSM-REA)}

The CSM-REA process, shown in Figure 2, is applied at the beginning of railway change projects to ensure that all applicable hazards are identified and managed. Risk management frameworks like this are required to fulfill obligations under the law, hence are quite well known in the Dutch railway context. The CSM-REA considers railway system safety from a systems perspective. It includes technical, operational, or organizational changes, which could impact the operating conditions of the railway system [13].

These regulations aim to implement change safely within the railway system by using a structured standardized process for identification and evaluation which includes [14]:

- Identifying what the change entails with respect to objective, boundary, scope, elements \& functions, interfaces.

- Determining and classifying hazards.

- Identifying the magnitude of identified hazards.

- Requirements for managing hazards effectively.

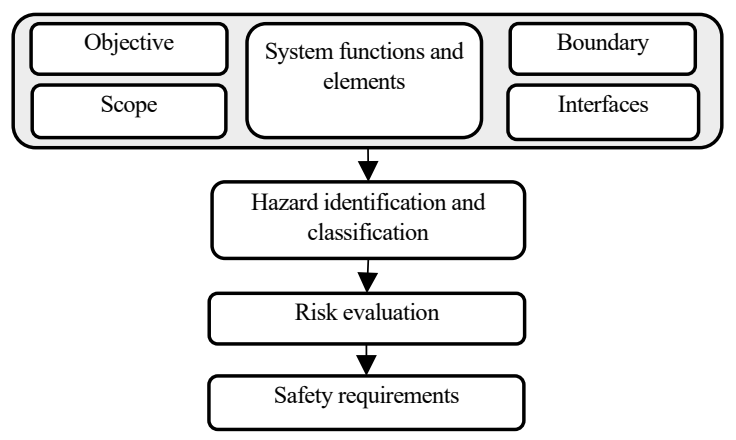

Figure 2: CSM-REA process adopted from [13].

Because SI is aimed at improving system performance by integrating changes in the existing railway system, the CSM-REA framework seemed fitting by broadening its scope from the safety perspective to a SI perspective i.e., using a structured process for code mapping.

Mapping codes on this framework, however, showed that a significant amount of the identified SI challenges did not necessarily fit one category, but could be fit onto multiple categories, as will be explained in Section 3. Additionally, the CSM-REA focuses mainly on evaluation not necessarily on planning and control [15].
To upgrade railway system performance, different changes are required to be integrated, requiring purposeful influencing/controlling of part of an organization or system. This led to the second framework by De Leeuw [12], which takes a systems perspective on conditions for effective control.

\subsection{Conditions for Effective Control (CEC)}

De Leeuw [12] describes five conditions for effective control of systems, from a systems viewpoint. This approach pays attention to problem orientation, thinking in contexts and processes, and thinking in systems and environments. The conditions of this framework are based on the idea that a system is to be brought or kept in its desired state, which is precisely the case in railways. He states that effective control of a system is not possible until the conditions in Table 1 are met [12].

Table 1: Five conditions for effective control from [12].

\begin{tabular}{|c|c|}
\hline CEC step & Description \\
\hline Objective & $\begin{array}{l}\text { Without an objective i.e., without a clear } \\
\text { picture of the desired state of the system, it } \\
\text { is impossible to determine whether the } \\
\text { steering is effective. }\end{array}$ \\
\hline $\begin{array}{l}\text { Model of } \\
\text { controlled } \\
\text { system }\end{array}$ & $\begin{array}{l}\text { To be able to influence a system, hence, to } \\
\text { decide whether to take control measures, } \\
\text { one must be able to predict the effect of } \\
\text { measures. After all, a measure is chosen to } \\
\text { bring about a certain effect. This requires a } \\
\text { model that can approximately answer the } \\
\text { question "How will the state of the system } \\
\text { change if I take this measure?" }\end{array}$ \\
\hline $\begin{array}{l}\text { Information } \\
\text { about } \\
\text { environment \& } \\
\text { state of the } \\
\text { system }\end{array}$ & $\begin{array}{l}\text { The future state of the controlled system is } \\
\text { determined by the current state of the } \\
\text { system, changes in the environment of the } \\
\text { system, and the relationships between the } \\
\text { elements of the system. Control, therefore, } \\
\text { requires information about the state of the } \\
\text { system and its environment. }\end{array}$ \\
\hline $\begin{array}{l}\text { Sufficient } \\
\text { control } \\
\text { measures }\end{array}$ & $\begin{array}{l}\text { Influencing requires control measures; } \\
\text { instruments with which the state of the } \\
\text { system could be changed. In order to be able } \\
\text { to control effectively, the number of } \\
\text { available control measures must be in } \\
\text { reasonable proportion to the variety of } \\
\text { circumstances that can occur. }\end{array}$ \\
\hline $\begin{array}{l}\text { Information } \\
\text { processing } \\
\text { capacity }\end{array}$ & $\begin{array}{l}\text { It is necessary to transform incoming } \\
\text { information about the environment and state } \\
\text { with the help of the model and taking into } \\
\text { account the objective into an effective } \\
\text { measure. Hence information must be } \\
\text { processed, requiring sufficient information } \\
\text { processing capacity. }\end{array}$ \\
\hline
\end{tabular}


The data-driven results of these comprehensive analyses will be further elaborated in the next section.

\section{Results and Discussion}

Due to the amount of data available, different crosssections were made of the analyzed data. These crosssections are shown in Table 2.

Table 2: SI challenges faced in the Dutch railway context, through different cross-sections.

\begin{tabular}{ll}
\hline Cross-section & Description \\
\hline Code frequency & $\begin{array}{l}\text { Manual (iterative) data processing and } \\
\text { coding based on SI issues mentioned during } \\
\text { the interviews. }\end{array}$ \\
\hline $\begin{array}{l}\text { Mapping on } \\
\text { CSM framework }\end{array}$ & $\begin{array}{l}\text { Mapping the codes on the CSM framework } \\
\text { in Figure 2. }\end{array}$ \\
\hline $\begin{array}{l}\text { Mapping on } \\
\text { CEC framework }\end{array}$ & $\begin{array}{l}\text { Mapping the codes on the CEC framework } \\
\text { described in Table 1. }\end{array}$ \\
\hline
\end{tabular}

\subsection{SI challenges based on code frequency}

The analyzed data resulted in 34 codes, challenges, and improvement opportunities regarding SI in the Dutch railway context, depicted in Figure 3. Due to space limitations within this paper, only approximately $50 \%$ of the challenges identified based on code frequency (corresponding to 9 challenges) will be explained in detail in Table 3.

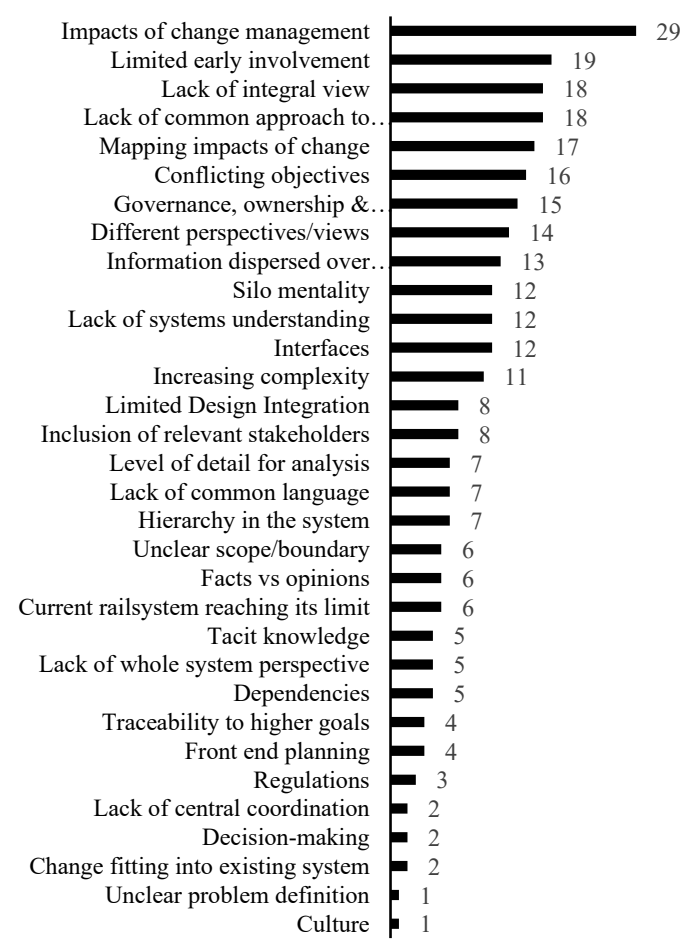

Figure 3: Code frequency of SI challenges.
Table 3: $50 \%$ of SI challenges based on code frequency.

\begin{tabular}{ll}
\hline Code & Description \\
\hline $\begin{array}{l}\text { Impacts of } \\
\text { change } \\
\text { management }\end{array}$ & $\begin{array}{l}\text { Challenges in managing the impacts of a } \\
\text { change. In event of a change, what does that } \\
\text { mean for the larger system? What other } \\
\text { parts, (sub)systems, organizations, } \\
\text { regulations, processes need to be taken into } \\
\text { consideration to facilitate smooth SI (of the } \\
\text { change). }\end{array}$ \\
\hline $\begin{array}{l}\text { Limited early } \\
\text { involvement }\end{array}$ & $\begin{array}{l}\text { The relevant stakeholders are not all } \\
\text { involved early on in the SI process. }\end{array}$ \\
\hline $\begin{array}{l}\text { Lack of } \\
\text { integral view }\end{array}$ & $\begin{array}{l}\text { Impacts of changes are not viewed } \\
\text { integrally: not viewed from a whole- } \\
\text { systems perspective. }\end{array}$ \\
\hline $\begin{array}{l}\text { Lack of } \\
\text { common } \\
\text { approach to } \\
\text { localize... }\end{array}$ & $\begin{array}{l}\text { There is no common, generally accepted } \\
\text { approach in this context to determine the } \\
\text { impacts of change. }\end{array}$ \\
\hline $\begin{array}{l}\text { Mapping } \\
\text { impacts of } \\
\text { change }\end{array}$ & $\begin{array}{l}\text { Creating insight in parts, (sub)systems, } \\
\text { organizations, regulations, processes } \\
\text { required to be taken into consideration to } \\
\text { facilitate smooth SI. }\end{array}$ \\
\hline $\begin{array}{l}\text { Conflicting } \\
\text { objectives }\end{array}$ & $\begin{array}{l}\text { Conflicting objectives can occur between } \\
\text { organizations. This certainly does not } \\
\text { encourage SI. }\end{array}$ \\
\hline organizations, how people look at the \\
system from their perspective. This could \\
alter goals and scope.
\end{tabular}

\subsection{Frameworks}

As stated in section 2, the codes were firstly mapped on the CSM-REA framework. The results of this mapping are shown in the Sankey diagram in Figure 4. Following this, the codes were mapped on the CEC framework, of which the results are shown in Figure 5. These diagrams indicate the relative frequencies: the distribution of coding across the framework categories concerning the infrastructure manager (ProRail) and main railway operator (NS).

\subsubsection{Mapping on CSM-REA}

The mapping in Figure 4 shows that Scope, Interfaces, and Boundary are among the largest identified code groups influencing SI. This indicates that SI is affected by difficulties identifying what exactly the change is (Figure 2). Interestingly, this mapping also shows that numerous challenges were identified which did not necessarily fit one on one onto the framework. During the mapping process, it became apparent that a significant portion (35\%) of the codes i.e., 'limited early involvement', 'silo mentality', and 
'lack of common language', covered all topics in the framework. Hence, were grouped as Overarching.

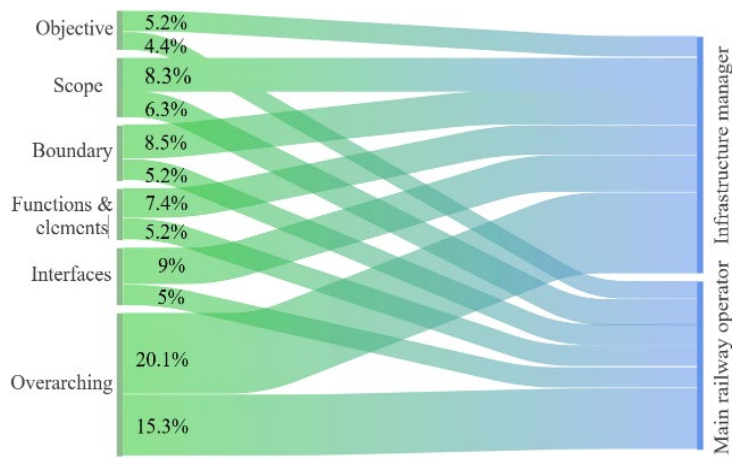

Figure 4: SI challenges mapped on the CSM-REA framework, indicating relative frequencies.

Because Overarching appeared to be the largest code group after mapping on this framework, limited conclusions could be drawn from this framework. For this reason and, because this framework focuses mainly on evaluation not necessarily on planning and control, the codes were mapped on the CEC framework.

\subsubsection{Mapping on CEC framework}

Mapping the codes on the CEC framework resulted in the Sankey diagram in Figure 5. All challenges could be mapped on this framework, except for one: 'Culture' which accounts for $0.17 \%$ of the identified challenges.

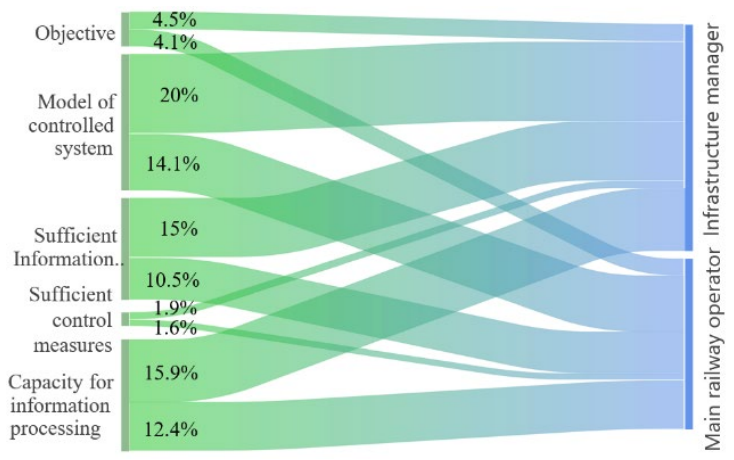

Figure 5: SI challenges mapped on the CEC framework, indicating relative frequencies.

This figure allows us to interpret the challenges from another angle:

1. Objective: (1) Conflicting objectives between organizations, (2) silo mentality, and (3) different views/perspectives.

2. Model of the controlled system: In this case of the railway system as a whole, but also of changes required to be implemented. This is among others due to: (1) increasing system complexity, (2) silo mentality, (3) lack of common language, (4) lack of common, integral approach to mapping impacts of changes in a complex context.

3. Information concerning the environment and state of the system: This is due to similar factors indicated in the previous point. In addition, limited early stakeholder involvement and interfaces/interdependencies are also mentioned as challenges in this group.

4. Sufficient control measures: A lack of control measures is indicated as the smallest SI challenge. This could mean, that according to interviewees, increasing control measures would not necessarily aid in managing SI in the Dutch railway sector. Another hypothesis is that the interviewees do not have the desire or ability to control the system as the pre-requirement on having a model of what to control, is limited.

5. Information processing capacity: This is impeded by (1) information dispersion across stakeholders, (2) lack of common, integral approach to mapping impacts of changes in a complex context, (3) governance, ownership \& responsibilities crossing blurred boundaries and, lack of systems understanding.

Based on this mapping, the following 3 factors from the CEC framework best matched SI challenges: (1) a model of the controlled system, (2) information processing capacity and, (3) Information concerning the environment and state of the system. Moreover, the Sankey diagram also shows that the mapped results from the main railway operator and the infrastructure manager differ percentages from each other, hence are more or less comparable.

\section{Conclusion \& future research}

As systems continue to grow in scale and complexity, managing SI has become a key concern. SI can be seen as 'a means to an end' that supports appropriate communication between technological, human and organizational elements, aimed at improving system performance. Consequently, the challenges related to SI can be similarly broad, varied, and of growing concern to railway organizations.

In order to investigate these challenges in the context of the Dutch railway sector, a series of interviews was carried out at the infrastructure managing- and main railway operating organization. Among the most frequent codes from the interviews were (1) 'Impacts of change management', (2) 'Limited early involvement', and (3) 'Lack of integral view'. This indicates the need for an SI overview and the need for actively managing SI challenges.

Mapping the challenges on both the CSM-REA and the CEC framework showed that the mappings to the 
infrastructure manager in comparison to the main railway operator are more or less comparable.

The mapping on the CSM-REA framework revealed that Scope, Interfaces, and Boundary are among the largest identified code groups influencing SI. This indicates that like CSM-REA, SI challenges also depend on clear objectives, boundaries, scopes, elements \& functions, and interfaces. However, not all SI challenges fit neatly onto the CSM-REA framework leaving a significant part of the codes classified as Overarching. Hence, another perspective on the challenges was provided by looking at the CEC framework.

Except for one code ('Culture'), all determined challenges could be mapped on the CEC framework. This indicates that SI also depends on (1) model of the controlled system, (2) information concerning the environment $\&$ state of the system, (3) sufficient information processing capacity, (4) sufficient control measures, and (5) objective, to get and/or keep systems in its desired state. For the Dutch railway system, the first three of these factors best matched the identified SI challenges. The results indicate that a model of the railway system and of changes required to be implemented are considered to be a prerequisite for effective system control. If such a model is lacking or cannot indicate changed system states, the influence and control of these systems will be challenging at the least. This integration challenge is not only a challenge for the individual railway stakeholders, but it can also hinder the overall aim of achieving better overall railway system performance at the (inter)national level.

In conclusion, most of the discussed SI challenges are concerned with (i) effectively determining what exactly is being changed, (ii) the scope of this change, and (iii) how this change would fit within the existing railway system. The existence of these challenges may hinder the effective control of this system, especially considering that it is becoming increasingly complex. Further research is therefore required to help practitioners and researchers recognize and resolve the underlying SI challenges.

\section{Future research}

Based on the research conducted in this paper, several opportunities for future research are proposed: (1) For each of the identified challenges, e.g., impacts of change management in complex railway context, further research could aid in trying to resolve these. (2) Determining causal relationships (and loops) between the codes, can help identify reinforcing and balancing loops. This could help indicate dynamic behavior in the Dutch railway sector.

\section{Acknowledgments}

This research is co-financed from the Research and Innovation contribution (PPP) from the Dutch Ministry of Economic Affairs and Climate. The authors acknowledge the support of the NS and ProRail, making this research possible through the framework of the SIRA project.

\section{References}

[1] Dumolo RN. Application of Systems Engineering to Railway Projects. Vol 3.; 2007.

[2] Rajabalinejad M, Frunt L, Klinkers J, van Dongen LAM. Systems Integration for Railways Advancement. In: Transportation Systems. Springer; 2019:27-40.

[3] Rajabalinejad M, Van Dongen LAM. Framing success: the Netherlands railways experience. Int J Syst Syst Eng. 2018;8(4):313.

[4] Sage AP, Lynch CL. Systems integration and architecting: An overview of principles, practices, and perspectives. Syst Eng. 1998;1(3):176-227.

[5] International Standardization Organization. NENISO/IEC/IEEE 15288 Systems and Software Engineering System Life Cycle Processes. Nederlands Normalisatieinstituut; 2015.

[6] Madni AM, Sievers M. Systems integration: Key perspectives, experiences, and challenges. Syst Eng. 2010;14(3):305-326

[7] Madni AM, Sievers M. System of systems integration: Key considerations and challenges. Syst Eng. 2014;17(3):330347.

[8] Rajabalinejad M. System Integration: Challenges and Opportunities. 2018:6-11.

[9] Leijten M, Koppenjan JFM. Asset management for the dutch railway infrastructure. In: International Conference on Next Generation Infrastructure Systems for Eco-Cities. Shenzhen; 2010.

[10] Association of European Railway Industries (UNIFE). InteGRrail-Intelligent Integration of Railway Systems.; 2010.

[11] Jovicic D, Officer P, Unit S. Guide for the application of the Commission Regulation on the adoption of a common safety method on risk evaluation and assessment as referred to in Article 6(3)(a) of the Railway Safety Directive. 2009;33(0):154

[12] Leeuw ACJ de. Bedrijfskundig management. Primair proces, Strateg en Organ Assen, K Van Gorcum. 2002.

[13] Rail Safety \& Standards Board. Guidance on the Common Safety Method for Risk Evaluation and Assessment Guidance on the Common Safety Method for Risk Evaluation and Assessment. GEGN8646. 2017.

[14] Ramtahalsing M, Jafari M, Braaksma J, Rajabalinejad M, Van Dongen L. The System (of Interest) Definitions phase: Key features and challenges in the Dutch Railway system. SOSE 2020 - IEEE 15th Int Conf Syst Syst Eng Proc. 2020:229-234.

[15] Maylor H, Turner N. Understand, reduce, respond: project complexity management theory and practice. Int J Oper Prod Manag. 2017;37(8):1076-1093. 